\title{
Analysis of a Public Key Approach Based on Polynomial Substitution
}

\author{
Harriet Fell \\ Northeastern University \\ Boston, Massachusetts \\ Whitfield Diffie \\ Bell-Northern Research \\ Mountain View, California
}

\section{Introduction}

Ever since the discovery of public key cryptography in $1975^{2}$, the search for public key cryptosystems has been a central theme of cryptographic research. The public key cryptosystems ${ }^{5,8,7}$ that have been investigated during this period, bowever, are slower than conventional systems, re quire more storage, and, being based on areas of mathematics that were not previously important in cryptography, have not inspired the same degree of trust as conventional systems. It would therefore be desirable to develop new techniques based on principles both different from those employed in current public key cryptosystems and more closely allied with conventional cryptography.

Several years ago, after the development of the public key concept, but before any plausable examples were known, a suggestion along these lines was made to one of the authors by John McCarthy of Stanford University, who said he had gotten the idea from talking with an algebraic geometer about birational transformations. The idea was to build inverse pairs of multivariate polynomial transformations by a procedure commonly employed in algebraic geometry to construct inverse pairs of rational transformations.

\section{The Fundamental Scheme}

Our approach is to regard the plaintext as an $n$-vector of elements selected from a suitable ring $R$ and build an invertible polynomial transformation $\mathrm{P}$ of several variables from $R^{n}$ to $R^{n}$. The coefficients of this transformation will be the public key and the inverse transformation $Q$ the secret key. Thus:

$$
\begin{array}{ccc}
\text { Plaintext } & \text { P } & \text { Ciphertext } \\
\bar{x}=\left(x_{1}, \ldots, x_{n}\right) & \mapsto & \left(P_{1}(\bar{x}), \ldots, P_{n}(\bar{x})\right)
\end{array}
$$

where $x_{1}, \ldots, x_{n} \in R$ and $P_{1}, \ldots, P_{n}$ are multivariate polynomials with coefficients in $R$.

Assume, for example, that the plaintext is a vector of three components, $x, y$ and $z$, from a ring $R$ and that $p_{0}$ and $p_{1}$ are polynomials each in one variable over $R$. We can now build up a polynomial transformation of three variables by acting on the variables one at a time.

In the first round, $(x, y, z)$ is carried to:

$$
\left(x_{1}, y_{1}, z_{1}\right)=(x, y, z+p(x, y)) .
$$

where $p$ can be either $p_{0}$ or $p_{1}$. In the second, $\left(x_{1}, y_{1}, z_{1}\right)$ goes to:

$$
\left(x_{2}, y_{2}, z_{2}\right)=\left(x_{1}+p\left(y_{1}, z_{1}\right), y_{1}, z_{1}\right)
$$


The process continues:

$$
\left(x_{3}, y_{3}, z_{3}\right)=\left(x_{2}, y_{2}+p\left(z_{2}, z_{2}\right), z_{2}\right)
$$

until after a number of rounds:

$$
P(x, y, z)=\left(x_{k-1}+p\left(y_{k-1}, z_{k-1}\right), y_{k-1}, z_{k-1}\right)
$$

is a nonlinear, invertible, polynomial transformation on a module, $M$, of dimension 3 over $R$. The secret key is the sequence of choices of $p_{0}$ or $p_{1}$ and the order in which they are applied to $x, y$, and $z$. For example:

$$
\begin{aligned}
\left(x_{1}, y_{1}, z_{1}\right) & =\left(x+p_{1}(y, z), y_{1} z\right) \\
& \left(x_{2}, y_{2}, z_{2}\right)=\left(x_{1}, y_{1}, z_{1}+p_{0}\left(x_{1}, y_{1}\right)\right) \\
& \left(x_{3}, y_{3}, z_{3}\right)=\left(x_{2}, y_{2}+p_{0}\left(x_{2}, z_{2}\right), z_{2}\right) \\
\left(x_{4}, y_{4}, z_{4}\right) & =\left(x_{3}, y_{3}, z_{3}++p_{1}\left(x_{3}, y_{3}\right)\right) \\
P(x, y, z)=\left(x_{5}, y_{5}, z_{5}\right) & =\left(x_{4}+p_{0}\left(y_{4}, z_{4}\right), y_{4}, z_{4}\right)
\end{aligned}
$$

The secret key is $((x, 1),(z, 0),(y, 0),(z, 1),(x, 0))$. The inverse transformation can be found by using the key in reverse: $((x, 0),(z, 1),(y, 0),(z, 0),(x, 1))$, i.e.:

$$
\begin{aligned}
\left(x_{4}, y_{4}, z_{4}\right)= & \left(x_{5}-p_{0}\left(y_{5}, z_{5}\right), y_{5}, z_{5}\right) \\
\left(x_{3}, y_{3}, z_{3}\right)= & \left(x_{4}, y_{4}, z_{4}-p_{1}\left(x_{4}, y_{4}\right)\right) \\
& \text { and so on. }
\end{aligned}
$$

Naturally the number of polynomials need not be limited to 2 ncr the dimension, $d$, of $M$ over $R$ to 3 , but there is probably no virtue in using polynomials of degree other than $d-1$.

This plan offers on its face, not only plausible hope for constructing inverse pairs of transformations, but one with very close ties to the shift register mathematics of conventional cryptography. The alternate transformation of variables $x$ and $y$ is closely analogous to alternate operation on the left and right halves in $\mathrm{DES}^{3}$. In general, the notion of modifying some components of a vector by adding to them functions of other components underlies all shift registers both linear and nonlinear ${ }^{1,4}$.

A key difference between the construction of conventional cryptosystems and public key cryptosystems lies in the way the systems are presented to the user. A shift register cryptosystem is a description of the way in which the plaintext is modified incrementally throught a number of interations to become the ciphertext. Such a description precludes public key use because it can equally readily be read in the other direction as a description of how to derive the plaintext from the ciphertext by incremental modifications. In order to develop a public key system along these lines, it is necessary to simplify the equations that arise from the incremental substitution process in such a way as to conceal the substitutions.

On first glance, it seems sufficient to carry out the substitutions as the process goes on. On second, it becomes obvious that the number of coefficients in the polynomials will grow to astronomical proportious after only a few interations. In order to prevent the equations from exploding into unusable bulk, some device must be found for eliminating most of the terms; the most obvious such devices are nilpotence and J-rings and these will be examined in the remainder of this paper.

\section{Reducing the Number of Coefficients}

\subsection{Nilpotence}

A ring $R$ is nilpotent if there is an integer $k \geq 1$ such that $(R)^{k}=\{0\}$. That means that for any elements $r_{1}, \ldots, r_{k} \in R$ the product $r_{1} r_{2} \cdots r_{k}$ is zero, so a multivariate polynomial with coefficients in $R$ can only have meaningful terms whose total degree does not exceed $k$. 
There is a hitch, however, in applying nilpotence in our fundamental scheme. We expect the scheme, described in section 2, to yield a transformation of the form:

$$
\begin{array}{ccc}
\text { plaintext } & \mathbf{P} & \text { ciphertext } \\
\bar{x}=\left(x_{1}, \ldots, x_{n}\right) & \mapsto & \left(P_{1}(\bar{x}), \ldots, P_{n}(\bar{x})\right)
\end{array}
$$

where $x_{1}, \ldots, x_{n} \in R$ and $P_{1}, \ldots, P_{n}$ bave coefficients in $R$. Note that no transformation of this form can be invertible. Suppose that each $P_{i}$ has constant term $C_{i}$. Let $T_{i}(\bar{x})=P_{i}(\bar{x})-C_{i}$. The system $\mathbf{P}$ is invertible if and only if the system $T=\left(T_{i}, \ldots, T_{n}\right)$ is invertible but $T$ can never be invertible as $T_{i}(\bar{x}) \in(R)^{2} \zeta R$.

What went wrong is that although the iteration in section 2 always produces an invertible transformation, if we apply this iteration scheme in a ring without a unit we do not get a transformation of the form (3.1) but rather one of the form:

$$
\begin{array}{ccc}
\text { plaintext } & \text { P } & \text { ciphertext } \\
\bar{x}=\left(x_{1}, \ldots, x_{n}\right) & \mapsto & \bar{x}+\left(P_{1}(\bar{x}), \ldots, P_{n}(\bar{x})\right)
\end{array}
$$

Where $x_{1}, \ldots, x_{n} \in R$ and $P_{1}, \ldots, P_{n}$ have coefficients in $R$. The transformation (3.1) is a polynomial transformation but its coefficients are not all in $R$ as $R$ is nilpotent and cannot contain a unit element.

We can still make use of nilpotence, however. We take $R$ to be a finite local ring, that is a finite commutative ring with 1 that has a unique maximal ideal $N$ of nilpotents. (Note $R / N$ is a Gnite feld.) The general form of the encryption transformation again becomes:

$$
\begin{array}{ccc}
\text { plaintext } & \mathbf{P} & \text { ciphertext } \\
\bar{x}=\left(x_{1}, \ldots, x_{n}\right) & \mapsto & \left(P_{1}(\bar{x}), \ldots, P_{n}(\bar{x})\right)
\end{array}
$$

where $x_{1}, \ldots, x_{n}$ and $P_{1}(\bar{x}), \ldots, P_{n}(\bar{x})$ all lie in $N$. That is, $\mathbf{P}$ is a multivariate polynomial transformation from $F^{n}$ to $R^{n}$ that is invariant and invertible on $N^{n}$. The number of coefficients in the polynomials is restricted by the nilpotence because terms of high total degree are identically zero on $N^{n}$ and we do not care how they behave on the rest of $R^{n}$. The transformations generated by the iteration scheme of section 2 will be of this type if we choose the polynomials $p_{1}, p_{2}, \ldots$ to have coefficients in $N$ or to have coefficients in $R$ but constant terms in $N$.

\section{$3.2 J$-Rings}

Let $R$ be a finite commutative ring. $R$ is a J-ring if there is an integer $d \geq 1$ such that $a^{d}=a$ for any $a \in R$. A multivariate polynomial over a J-ring can be reduced to a polynomial all of whose terms have individual degrees $\leq d$ (or total degrees $<d n$ where $n$ is the number of variables). This time the transformation will be of the form of equation (3.1).

\subsection{Upper. Triangular Matrices}

We represent the plaintext by a pair of upper-triangular matrices with entries from a finite ring $R$. The encryption transformation will be of the form:

$$
\begin{array}{ccc}
\text { plaintext } & \mathbf{P} & \text { ciphertext } \\
\left(X_{1}, X_{2}\right) & \rightarrow & \left(P_{1}\left(X_{1}, X_{2}\right), P_{2}\left(\mathrm{~K}_{1}^{-}, X_{2}\right)\right)
\end{array}
$$

where $X_{1}, X_{2}$ are upper-triangular $k \times k$ matrices over $R$ and $P_{1}, P_{2}$ bave coefficients in $R$. If $M_{1}, \ldots, M_{k}$ are upper triangular $k \times k$ matrices over $R$ then the product $M_{1} \cdots M_{k}$ is zero. This means that the polynomials will have terms of total degree at most $k-1$. As the matrices do not commute, there are more terms to deal with than in the commutative nilpotent case (3.1) but there is also hope that non-commutativity will make lower degree polynomial systems more difficult to invert. 


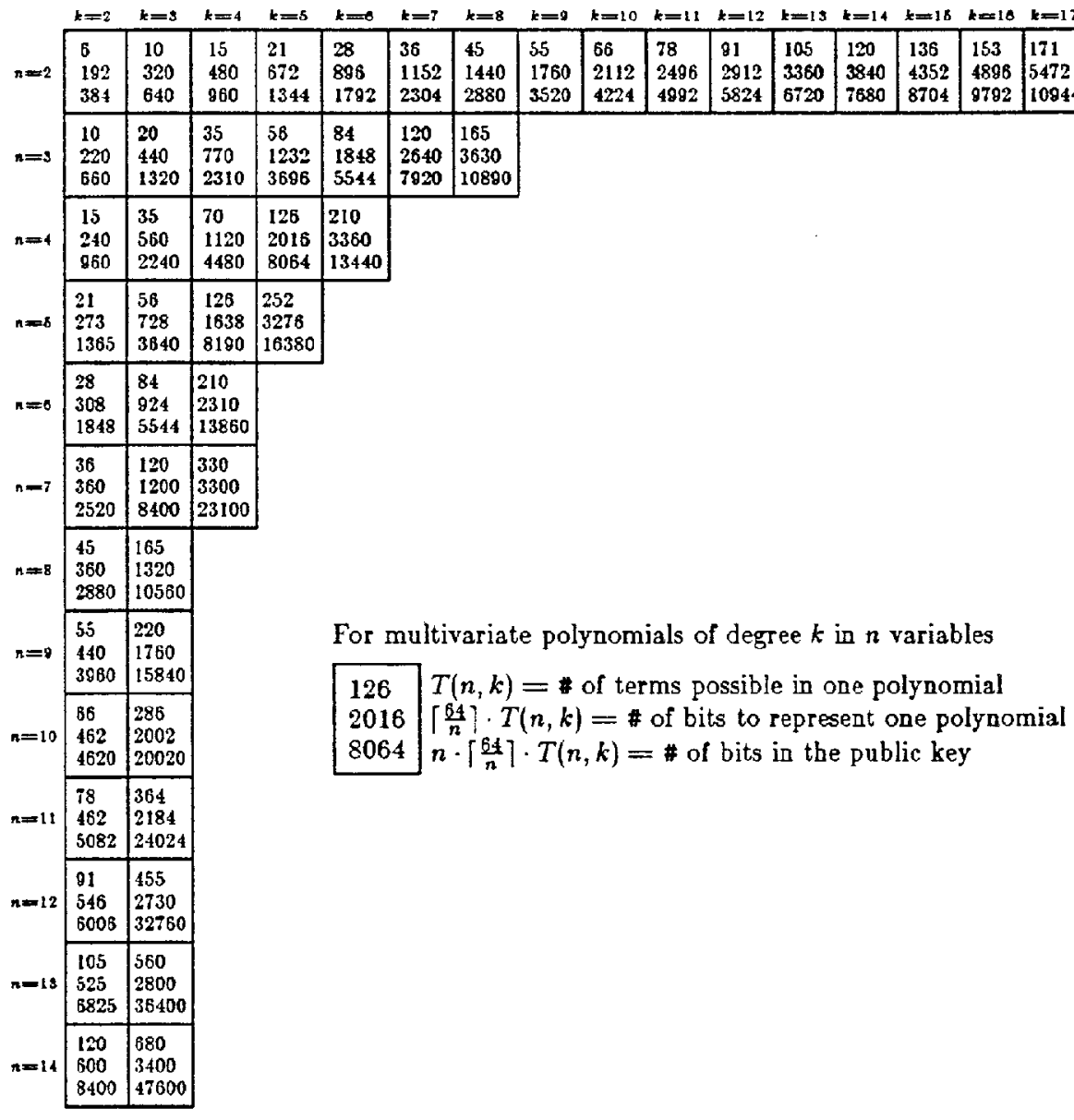

Figure 3.1 Bits of Key in Commutative Case (Plaintext $=64$ bits)

\section{Finding Systems of Practical Size}

The public key in the system we have proposed consists of the coefficients of the polynomials making up the transformation $P$. We do not want a key that is too large and have taken 10,000 bits to be the upper limit on the size of key that we will consider.

\subsection{The Commutative Case}

We must first count the maximum number of terms in a polynomial of total degree $k$ in $n$ variables. This number, $T(n, k)$ can be computed recursively as follows:

$$
\begin{array}{ll}
T(1, k)=k+1 & \text { (i.e., } \left.1, X, \ldots, X^{k}\right) \\
T(n, 1)=n+1 & \text { (i.e., } \left.1, X_{1}, \ldots, X_{n}\right) \\
T(n, k)=T(n, k-1)+T(n-1, k) & \forall_{k, n>1} .
\end{array}
$$




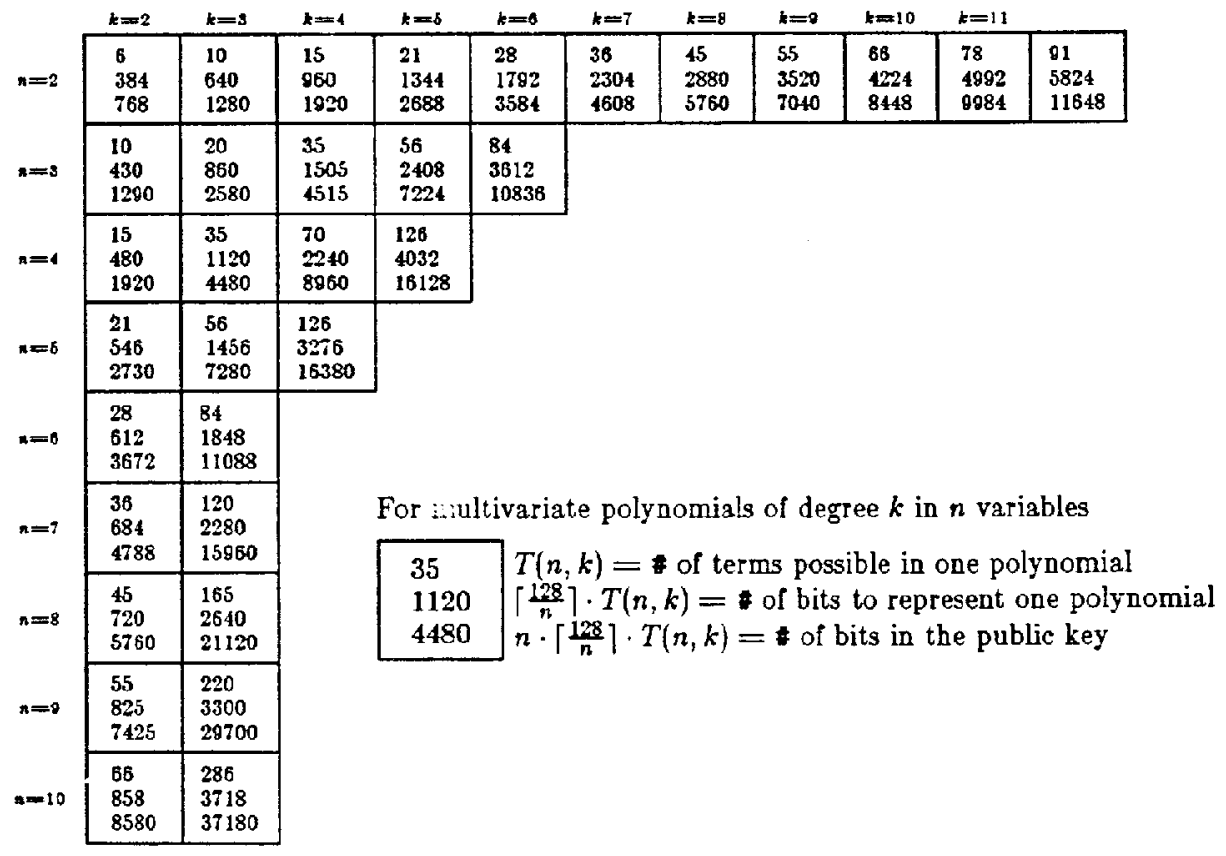

Figure 4.2 Bits of Key in Commutative Case (Plaintext $=128$ bits)

The recursion step follows since $T(n, k-1)$ is the number of terms of total $\leq k$ in which $X_{n}$ appears. Each such term is of the form $X_{n}$ times a term in n variables with total degree $\leq k-1$. $T(n-1, k)$ is the number of terms of total degree $\leq k$ in which $X_{n}$ does not appear.

Now we need the number of bits necessary to represent the coefficients of a polynomial of total degree $k$ in $n$ variables. This clearly depends on the size of the ring but there are restrictions on the ring size if we want the plaintext size to conform to present standards. If the plaintext $\left(X_{1}, \ldots, X_{n}\right)$ is to have 64 bits then each $X_{i}$ must represent $\left[\frac{84}{n}\right]$ bits. If $R$ is a J-ring so that encryption method (3.1) is used, then $R$ must have cardinality $2^{\left[\frac{\Delta 4}{n}\right]}$. The number of bits needed to represent a single polynomial of total degree $k$ in $n$ variables is given by $\left[\frac{64}{n}\right\rceil \cdot T(n, k)$. The number of bits in the public key polynomial transformation is $n \cdot\left[\frac{64}{n}\right] \cdot T(n, k)$. These numbers are computed and presented in Figure 4.1. The same computation for a plaintext of 128 bits is presented in Figure 4.2.

If the ring is local and the general encryption method of equation 3.3 is used, then the cardinality of the nilpotent ideal, $N$, must be $2^{\left[\frac{04}{n}\right]}$ or $2^{\left[\frac{128}{n}\right]}$. The cardinality of $R$ is at least twice that of $N$. The number of bits needed to represent the public key is at least double the numbers that appear in Figures 4.1 and 4.2. If the specific iteration described in section 2 is used with $P_{1}, \ldots, P_{n}$ having coefficients in $N$ then the number of bits needed to represent the public key is exactly as shown in Figures 4.1 and 4.2.

It is striking that if we are restricted to 10,000 bits of key then the polynomials making up the encryption transformation and also those making up its inverse can have no more than 153 terms ( $n=2, k=16,64$-bit plaintext). We shall see in the next section that this is too small for cryptographic security. 


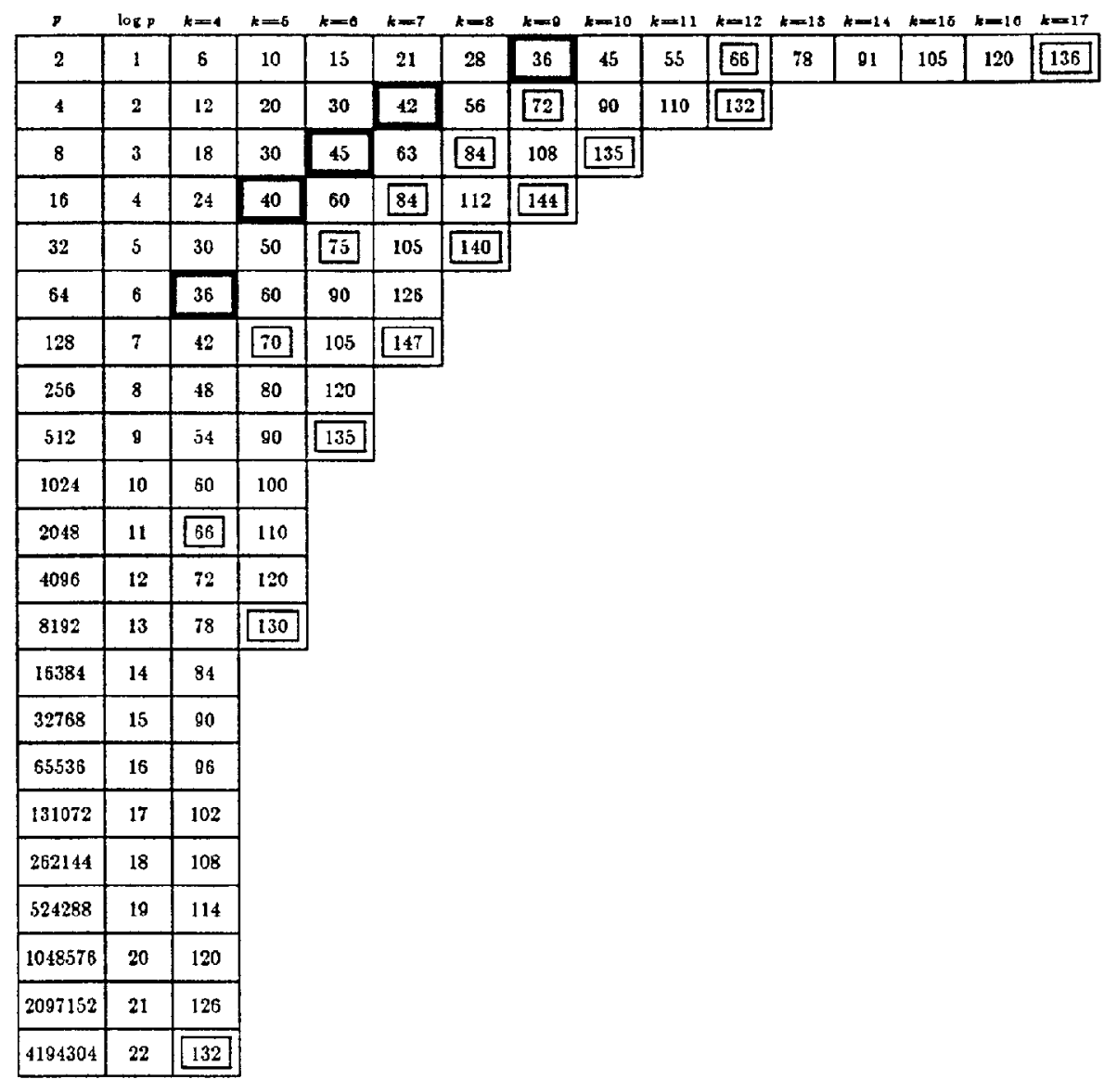

Figure 4.3 Bits of Plaintext in ove Upper-Triangular Matrix over $Z_{p}$

\subsection{The Upper-Triangular Case}

We consider the special case of $k \times k$ upper-triaggular matrices over $Z_{p}$. We have taken $p$ to be a power of 2 so that there is no bit loss in representing the ring. A single matrix carries $(\log p)\left(\frac{k^{2}-k}{2}\right)$ bits. In Figure 4.3 we show the number of bits of plaintext in a pair of upper-triangular matrices over $Z_{p}$.

Figure 4.3 also provides information in the case of a general commutative ring $R$. The number of bits shown for a particular $p$ provides lower (upper) bounds when $R$ is any commutative ring with cardinality greater than (less than) $p$. 


\begin{tabular}{|c|c|c|c|c|c|c|}
\hline & & & bits per & $\begin{array}{r}2^{k}-1= \\
\text { terms per } \\
\text { matrix }\end{array}$ & $\begin{array}{r}\text { bits ol key }= \\
\text { bolyomial per } \\
\text { polynomial }\end{array}$ & $\begin{array}{r}\text { bits per } 2 \\
\text { polynomials }\end{array}$ \\
\hline
\end{tabular}

64 Bit Plaintext

\begin{tabular}{|c|c|c|c|c|c|c|}
\hline 2 & 1 & 9 & 36 & 511 & 511 & 1022 \\
\hline 4 & 2 & 7 & 42 & 127 & 254 & 508 \\
\hline 8 & 3 & 5 & 45 & 63 & 189 & 378 \\
\hline 16 & 4 & 5 & 40 & 31 & 124 & 248 \\
\hline 64 & 6 & 4 & 36 & 15 & 90 & 180 \\
\hline
\end{tabular}

128 Bit Plaintext

\begin{tabular}{|c|c|c|c|c|c|c|}
\hline 2 & 1 & 12 & 66 & 4095 & 4095 & 8180 \\
\hline 4 & 2 & 8 & 72 & 511 & 1022 & 2044 \\
\hline 8 & 3 & 8 & 84 & 255 & 765 & 1530 \\
\hline 16 & 4 & 7 & 84 & 127 & 508 & 1016 \\
\hline 32 & 5 & 6 & 75 & 63 & 315 & 630 \\
\hline 128 & 7 & 5 & 70 & 31 & 217 & 434 \\
\hline 2048 & 11 & 4 & 66 & 15 & 165 & 330 \\
\hline
\end{tabular}

Figure 4.4 Bits of Key; Upper-triangular Case

In Figure 4.4 we compute the number of bits in the public key when the plaintext has 64 and 128 bits respectively. To do this computatior; we must find the number of terms in a polynomial in 2 upper-triangular $k \times k$ matrices. Recall that these polynomials will have total degree at most $k-1$. The number of terms of exactly degree $j$ in 2 non-commuting variables is $2^{j}$. It is the same as making $j$ choices from 2 items with repetition allowed. The total number of terms is, therefore:

$$
\sum_{j=0}^{k-1} 2^{j}=2^{k}-1
$$

There appear to be cases where the key is not terribly big and where the number of terms in each polynomial is large enough that we might have cryptographic security. We will see, however, in the next section that we can solve for the coefficients of the polynomials in the inverse transformation in layers so that we need never face a very large system of equations. 


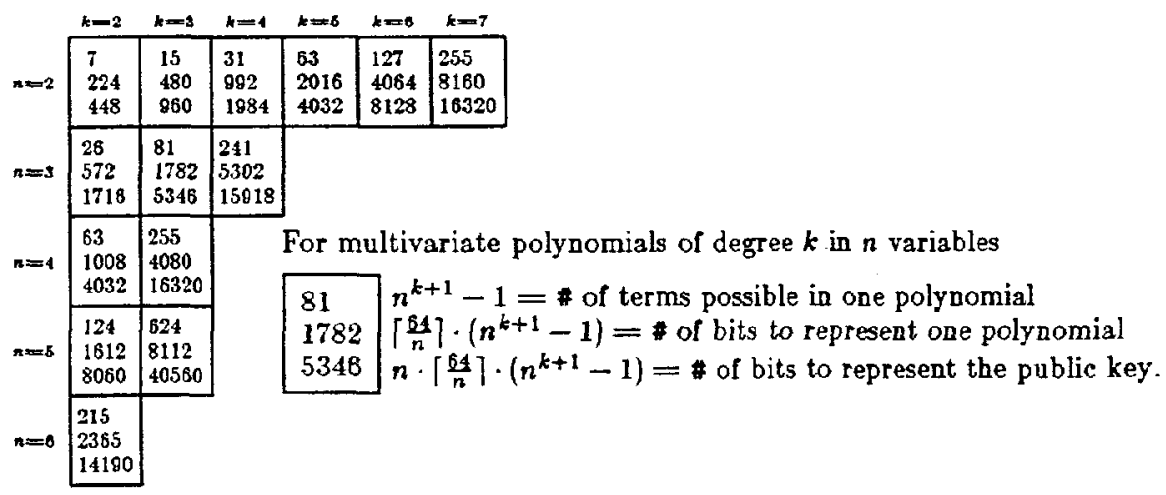

Figure 4.5 Bits of Key; Non-commutative Case; 64-Bit Plaintext

\subsection{The General Non-Commutalive Case}

As will be shown, in the next section, the commutative and upper-triangular cases are not cryptographically secure so we offer one other suggestion. Let $R$ be a non-commutative finite ring. In figure 4.5 we show the number of bits of key in this case. The number of terms in a polynomial of total degree $k$ in $n$ non-commuting variables is given by $n^{k+1}-1$. The reasoning is the same as that used to compute the number of terms in the polynomials of section 4.1 above. Figure 4.5 shows that there are very few cases to investigate. The number of terms in the polynomials is small but there is some hope that the complications of non-commutative arithmetic will impede cryptanalysis.

\section{Inverting These Systems}

Assume that we know the public key, $\mathbf{P}=\left(P_{1}, \ldots, P_{n}\right)$, and we want to find a transformation $\mathrm{Q}=\left(Q_{1}, \ldots, Q_{n}\right)$ such that $Q_{i}\left(P_{1}(\bar{x}), \ldots, P_{n}(\bar{x})\right)=X_{i}(i=1, \ldots, n)$. We know that such a system $Q$ of polynomials exists and that the $Q_{i}$ have the same types of terms as the $P j$. That is:

$$
Q_{i}=a_{i}+b_{i_{1}} V_{1}+\cdots+b_{i_{n}} V_{n}+c_{i 1_{2}} V_{i}^{2}+\cdots
$$

We know which terms are present, we must find the coefficients of $Q_{i}(i=1, \ldots, n)$.

\subsection{If $R$ is a $J$-ring}

Pick a vector $\bar{A}=\left(A_{1}, \ldots, A_{n}\right)$ in $R^{n}$. Compute $P_{1}(\bar{A}), \ldots, P_{n}(\bar{A})$. Set $Q_{1}\left(P_{1}(\bar{A}), \ldots, P_{n}(\bar{A})\right)=$ $A_{1}$. This gives a linear equation with coefficients in $R$ whose unknowns are the coefficients of $Q_{1}$. Let $q$ by the number of coefficients of $Q_{1}$. If we can produce vectors $\bar{A}_{i}=\left(A_{i_{1}}, \ldots, A_{i_{n}}\right)$ in $R^{n}(i=$ $1, \ldots, q)$ such that the resulting linear equations are independent then, hopefully, we can solve for the coefficients of $Q_{1}$. As $Q$ is invertible, we know that such an independent system exists. Any J-ring $R$ is a direct sum of finite fields ${ }^{8}$. Hence the system can be solved independently over each component field of $R$ using standard techniques of linear algebra over felds. A system of approximately 150 equations can be solved in a reasonable time by existing techniques and in our systems of practical size, $Q_{i}$ never has more than 153 coeficients.

There remains the problem of generating $q$ independent equations. We suggest the following simple procedure. 1. choose a $\bar{A} \not \bar{O}$ in $R_{n}$ and accept the linear equations it produces. 2. After 
haying found $k-1$ independent equations, choose a new vector $\bar{A} \not \equiv \bar{O}$ at random and accept it if it is independent of the $k-1$ vectors already found. Otherwise, discard it and repeat this step until you succeed. If, at each stage, the system is put, componentwise, into reduced row-echelon form, then checking the new equation and row-reducing the new system are both easy. We cannot prove that this method will produce the necessary $q$ equations in a reasonable amount of time but believe it does for the following reasons:

Assume $R=Z_{p}, p$ a prime. If $k$ vectors are chosen at random from $\left(Z_{p}\right)^{q}$ then the probability that they are independent is given by $\left(1-\frac{1}{p^{q}}\right)\left(1-\frac{1}{p^{q-1}}\right) \cdots\left(1-\frac{1}{p^{q-k+1}}\right)>\frac{p-2}{p-1}$ so the probability that a radom $q \times q$ determinant over $Z p$ is non-singular is given by:

$$
\prod_{i=1}^{q}\left(1-\frac{1}{p^{i}}\right)>\frac{p-2}{p-1} \text {. }
$$

Although this gives 0 as a lower bound when $P=2$ the products are actually greater than $1 / 4$ in this case.

Unfortunately, the coefficient vectors in these equations are not generated at random from all possible $p^{q}$ vectors over $Z_{p}$; we can only generate $p^{n}$ vectors but, expect that they will be randomly distributed in the larger set. Given this, the above argument shows that we are likely to generate $q$ independent equations without much difficulty.

\subsection{The Nilpotent Case}

The message is a vector in $N^{n}$ where $N$ is a nilpotent ring embedded as a maximal nilpotent ideal in a local ring $R$. The quotient ring $R / N$ is a finite field. $N^{n}$ is invariant under the public key polynomial map $\mathrm{P}: R^{n} \mapsto R^{n}$. That is, $\mathrm{P}: N^{n} \mapsto N^{n}$ is one to one and onto. The component polynomials $P_{i}$ of $P$ have coefficients in $R$. To find the coefficients of $Q=P^{-1}$ we first work over the field $R / N$ and then raise the solution to $R$.

We can assume that $P_{i}(i=1 \ldots n)$ has no constant term. Otherwise $\mathrm{P}(\bar{X})=\mathrm{T}(\bar{X})+\bar{C}$ where $\bar{C}$ is a constant vector in $N^{n}$ and $T(X)$ is a polynomial transformation whose components have no constant terms. $\mathrm{P}$ is invertible on $N^{n}$ if and only if $\mathrm{T}$ is. If $\mathrm{U}=\mathrm{T}^{-1}$ on $N^{n}$ then: $\mathbf{Q}(\bar{Y})=\mathbf{U}(\bar{Y}-\bar{C})$ is $\mathbf{P}^{-1}$ on $N$.

Let $P_{L}$ and $Q_{L}$ be the linear parts of $P$ and $Q$. Then

$$
\bar{x}=Q(P(\bar{x}))=Q_{L}\left(P_{L}(\bar{x})\right)+\text { higher order terms } .
$$

Let $\left(\mathbf{P}_{L} \bmod N\right)$ mean the polynomial obtained by replacing each coefficient, $c$, of $\mathbf{P}_{L}$ by $(C \bmod N)$. We invert $P_{L}$ to find $Q_{L}$. First find

$$
\mathrm{Q}_{L}=\left(\mathrm{P}_{L} \bmod N\right)^{-1} \quad \text { over } R / N
$$

Now form $Q_{L}^{\prime \prime}$ with coefficients in $R$ by replacing eacb coefficient of $Q_{L}^{\prime}$ by a representative in $R$ of its class. Then

$$
\mathrm{Q}_{L}^{\prime \prime} \circ \mathrm{P}_{L}=l+B \text { where } B \text { has eatries in } N
$$

and

$$
\left(I-B+B^{2}-\cdots \pm B^{k-1}\right) Q_{L}^{\prime \prime} P_{L}=I \pm B^{k}=I \text { on } N^{n}
$$

So set

$$
\mathrm{Q}_{L}=\mathrm{P}_{L}^{-1}=\left(I-B+B^{2}-\cdots \pm B^{k-1}\right) Q_{L}^{\prime \prime} .
$$


Now go on to the quadratic terms. Let $\mathbf{P}_{Q}$ and $\mathbf{Q}_{Q}$ be the quadratic parts and $\mathbf{P}_{H}$ and $\mathbf{Q}_{H}$ the higher order parts of $\mathbf{P}$ and $Q$ respectively. Then

$$
\begin{aligned}
\bar{x}=Q(P(\bar{x}))= & \mathbf{Q}_{L}\left(\mathbf{P}_{L}(\bar{x})+\mathbf{P}_{Q}(\bar{x})+\mathbf{P}_{H}(\bar{x})\right)+\mathbf{Q}_{Q}\left(\mathbf{P}_{L}(\bar{x})+\mathbf{P}_{Q}(\bar{x})+\mathbf{P}_{H}(\bar{x})\right) \\
& +\mathbf{Q}_{H}\left(\mathbf{P}_{L}(\bar{x})+\mathbf{P}_{Q}(\bar{x})+\mathbf{P}_{H}(\bar{x})\right) \\
= & \mathbf{Q}_{L}\left(\mathbf{P}_{H}(\bar{x})\right)+\mathbf{Q}_{L}\left(\mathbf{P}_{Q}(\bar{x})\right)+\mathbf{Q}_{Q}\left(\mathbf{P}_{L}(\bar{x})\right)+\text { higher order terms. }
\end{aligned}
$$

This gives a system of equations whose unknowns are the coefficients of $Q_{q}$. We can proceed as with the linear parts, finding the coefficients of $\mathbf{Q}$ one degree at a time.

For rings of practical size, these systems are therefore too easily solved to be secure.

\subsection{The Upper-Triangular Case}

The encrypting transformation is $\left(X_{1}, X_{2}\right) \mapsto \mathrm{P}\left(X_{1}, X_{2}\right)=\left(P_{1}\left(X_{1}, X_{2}\right), P_{2}\left(X_{1}, X_{2}\right)\right)$ where $X_{1}, X_{2}$ are upper-triangular matrices over a commutative ring $R$ and $P_{1}, P_{2}$ are polynomials with coefficients in $R$. To decrypt, we must find a polynomial system $Q=\left(Q_{1}, Q_{2}\right)$ such that $Q_{i}\left(\mathbf{P}\left(X_{1}, X_{2}\right)\right)=X_{i}(i=1,2)$. As before, we can use $\mathbf{P}$ and $\mathbf{Q}$ to produce pairs $(U, V), Q(U, V)$ and to set up a system of linear equations in the coefficients of $\mathbf{Q}$. This system is particularly easy to solve as only the linear and constant terms show up in the entries just above the diagonal of $\mathbf{Q}(U, V)$. The quadratic terms enter into the entries two levels above the diagonal and so on. We can, therefore, solve for the coefficients of $Q$ in a layered manner, similar to the nilpotent case.

\section{Conclusions}

We set out to build a public key cryptosystem by repeatedly substituting for variables in multivariate polynomials and simplifying the results to conceal the substitution process. There seems, however, to be no way to build such a system that is both secure and has a public key of practical size when the devices used to limit the number of coefficeints are nilpotence and J-rings. We have only shown, however, that it is impossible to produce such a system if the total degree of the encryption polynomial determines the size of the public key. Perhaps, by properly choosing $p_{0}$ and $p_{1}$, we can employ the fundamental scheme to produce sparse encrypting polynomials. Then the public key could be kept small while the encrypting polynomial bas large total degree and is difficult to invert.

\section{References}

[1] Don Coppersmith and Edna Grossman, "Generators for Certain Alternating Groups with Applications to Cryptography,"SIAMJ. Appl. Math., Vol. 28, No. 4, pp. 624-627, Dec 1975.

[2] Whitfield Diffie and Martin E. Hellman, "New Directions in Cryptography," IEEE Trans. Info. Thy., Vol. IT-22, No. 6, pp. 644-654, November 1976.

[3] Data Encryption Standard, FIPS Pub. No. 46, National Bureau of Standards, 15 January 1977.

[4] Solomon W. Golomb, Shift Register Sequences, Holden Day, San Francisco, 1967.

[5] R. McLeice, A Public-Key Cryptosystem Based On Albebraic Coding Theory, DSN Progress Report 42-44, Jet Propulsion Lab, Calif. Inst. of Tech., Pasadina CA, Jan-Feb 1978.

[6] R. C. Merkle and M. E. Hellman, "Hiding Information and Sigatures in Trapdoor Knapsacks," IEEE Transations on Information Theory, Vol. IT-24, No. 5, pp. 525-530, September 1978.

[7] R. L. Rivest, A. Shamir, and L. Adleman, "A Method for Obtaining Digital Signatures and Public Key Cryptosystems," CACM, Vol. 21, No. 2, pp. 120-126, February 1878.

[8] Gustavus J. Simmons. Personal Communication. 